\title{
Urinary proteome analysis enables assessment of renoprotective treatment in type 2 diabetic patients with microalbuminuria
}

\author{
Sten Andersen ${ }^{1}$, Harald Mischak ${ }^{2,3,6^{*}}$, Petra Zürbig ${ }^{3,6}$, Hans-Henrik Parving ${ }^{4,5}$, Peter Rossing ${ }^{1,6}$
}

\begin{abstract}
Background: Previously the angiotensin II receptor blocker Irbesartan has been demonstrated to reduce the risk for progression from microalbuminuria to macroalbuminuria in type 2 diabetic patients. The purpose of this study was to evaluate the effect of treatment with Irbesartan in type 2 diabetic patients with microalbuminuria on the urinary proteome.

Methods: High-resolution capillary-electrophoresis coupled to mass-spectrometry (CE-MS) was used to profile the low-molecular-weight proteome in urine of a subgroup of patients from a two year randomized irbesartan versus placebo therapy trial, which included hypertensive type 2 diabetic patients with microalbuminuria on ongoing antihypertensive medication (IRMA2-substudy).

Results: We demonstrate that the therapy with 300 mg Irbesartan daily over a period of two years results in significant changes of the urinary proteome. Both, a classifier developed previously that consists of urinary peptides indicative of chronic kidney disease, as well as several individual peptides changed significantly after treatment. These changes were not observed in the placebo-treated individuals. Most prominent are changes of urinary collagen fragments associated with progression of diabetic nephropathy, indicating normalization in urinary peptides.

Conclusion: CE-MS analysis of urine enabled identification of peptides as potential surrogate markers for renoprotection in microalbuminuric type 2 diabetic patients, which show persistent improvement after longterm treatment with Irbesartan. The results suggest that a major benefit of treatment by Irbesartan may be improvement of collagen turnover, reduction of fibrosis. They further suggest that urinary proteome analysis could be utilized to assess potential benefit of therapeutic intervention, providing statistically significant results even on a small population.
\end{abstract}

\section{Background}

At present more than 170 million people worldwide have diabetes and the number is expected to double within the next 20 years mainly due to an epidemic increase in the prevalence of type 2 diabetes [1]. Type 2 diabetes is associated with an increased occurrence of cardiovascular disease and approximately $40 \%$ of all diabetic patients are at risk of developing diabetic nephropathy which has become the leading cause of end-stage renal disease (ESRD) in the Western world [2].

\footnotetext{
* Correspondence: h.mischak@clinmed.gla.ac.uk

${ }^{2}$ BHF Glasgow Cardiovascular Research Centre, Glasgow, United Kingdom Full list of author information is available at the end of the article
}

Therefore, the early identification and subsequent endorgan protective treatment of all patients at risk for ESRD is of outmost importance. Patients with persistent microalbuminuria [urinary albumin excretion (UAE) between 30 and $300 \mathrm{mg} / 24$ hours] have a 10 to 20 times increased risk of developing diabetic nephropathy as compared to patients with normoalbuminuria [2]. In addition, the occurrence of microalbuminuria is associated with an increased risk of premature death due to cardiovascular disease [3].

Reduction of UAE by blockade of the renin-angiotensin-aldosterone system (RAAS) has emerged as a key treatment goal for both reno- and cardiovascular protection $[4,5]$. Data from the large clinical "Irbesartan in 
Patients with type 2 diabetes and Microalbuminuria" (IRMA2) study [6] firmly demonstrated that treatment with the angiotensin II receptor blocker (ARB) Irbesar$\tan , 300 \mathrm{mg}$ once daily, reduces UAE and the risk of progression to overt diabetic nephropathy in hypertensive patients with type 2 diabetes and persistent microalbuminuria. Furthermore, in type 2 diabetic patients with more advanced renal disease, ARBs have been shown to reduce the risk of reaching the combined renal end point of doubling in serum creatinine, ESRD, or death $[5,7]$. Since 2002, ARBs have consequently been recommended as first-line therapy in hypertensive type 2 diabetic patients with microalbuminuria or overt diabetic nephropathy according to guidelines from the American Diabetes Association [8].

Recently, we and others demonstrated that diabetic nephropathy and chronic renal disease in general are reflected by specific peptides and proteins in urine [9-24], and the human urinary proteome has been extensively investigated to gain insight about disease processes affecting the kidney and the urogenital tract [12,25-28]. Urinary proteins and peptides originate not only from glomerular filtration, but also from tubular secretion, epithelial cells shed from the kidney and urinary tract, secreted exosomes [29], and seminal secretions [30-32]. Thus, in principle, urine is a rich source of biomarkers for a wide range of diseases due to specific changes in its proteome [33-36]. Urine is a preferred body fluid for proteome analysis, as it is quite stable, probably due to the fact that it is "stored" for hours in the bladder, hence proteolytic degradation by endogenous proteases, a major obstacle in proteomics studies focusing on blood [37], may be essentially complete by the time of voiding $[38,39]$. This also enabled the establishment of human urine reference standard samples [40]. In pilot studies aiming toward differential diagnosis of certain types of CKD we could show that several peptides are differentially excreted in the urine of patients with different chronic kidney diseases compared to healthy individuals [41,42]. An optimized protocol for sample preparation and analysis has been developed, that includes removal of proteins above $25 \mathrm{kDa}$ without significant loss of low-molecular-weight urinary components [43]. Using this protocol, urinary biomarkers enabling differential diagnosis of specific single chronic renal diseases (IgA nephropathy, diabetic nephropathy, and ANCA-associated vasculitis) with good sensitivity and specificity in blinded data-sets could be identified $[13,19,21,44]$. Employing previously established biomarkers and biomarker patterns as classifiers [19,20], we investigated if a therapeutic benefit of Irbesartan in microalbuminuric type 2 diabetes patients can by displayed by proteomic changes in urine. In addition, we aimed at identifying those peptides that show significant changes upon Irbesartan treatment, as these may reveal further insights into the pathophysiology of disease, and allow assessment of therapeutic efficacy.

\section{Methods}

\section{Patient characteristics}

Spontaneously voided urine samples were collected from type 2 diabetic patients followed at Steno Diabetes Center as a subset of the 'IRMA2' study described previously [45]. The study was in compliance with the Helsinki Declaration and all patients gave written informed consent. The study was approved by the ethics committee of Copenhagen County KA 97015 gms. Samples from all patients included in the study receiving either Irbesartan in a dose of $300 \mathrm{mg}$ once daily or placebo were employed for CE-MS analysis, if samples were available from both, baseline and after two years of treatment. As the effect of a dose of $150 \mathrm{mg}$ once daily was not significant on UAER, in the IRMA2 study, we only used $300 \mathrm{mg}$ daily and compared with placebo. In total, samples from 22 patients (11 irbesartan and 11 placebo) were available. At baseline 2 patients in the placebo and 4 in the irbesartan group were treated with insulin, after 2 years it was 5 and 4 . Unchanged throughout the study, 8 patients in the placebo and 6 in the irbesartan group were treated with oral hypoglycemic agents at baseline, 3 patients in each group were treated with a statin at baseline, 8 patients in the placebo and 5 in the irbesartan group were treated with aspirin for cardiovascular protection at baseline. Demographic data of the patients included are shown in Additional file 1, spreadsheet: 'patient data'.

\section{Sample preparation}

Samples consisted of overnight urines, stored in aliquots at $-20^{\circ} \mathrm{C}$ for $8-12$ years, which were prepared essentially as described [46]. A $0.7 \mathrm{~mL}$ aliquot was thawed immediately before use and diluted with $0.7 \mathrm{~mL} 2 \mathrm{M}$ urea, 10 $\mathrm{mM} \mathrm{NH} \mathrm{NH}_{4} \mathrm{OH}$ containing $0.02 \%$ SDS. In order to remove high molecular weight polypeptides, samples were filtered using Centrisart ultracentrifugation filter devices (20 kDa molecular weight cut-off; Sartorius, Goettingen, Germany) at 3,000 g until $1.1 \mathrm{~mL}$ of filtrate was obtained. Subsequently, filtrate was desalted using PD-10 column (GE Healthcare, Sweden) equilibrated in $0.01 \% \mathrm{NH}_{4} \mathrm{OH}$ in $\mathrm{HPLC}$-grade water. Finally, samples were lyophilized and stored at $4{ }^{\circ} \mathrm{C}$. This procedure results in an average recovery of sample in the preparation procedure $\sim 85 \%$ [21]. Shortly before CE-MS analysis, lyophilisates were resuspended in HPLC-grade water to a final protein concentration of $0.8 \mu \mathrm{g} / \mu \mathrm{L}$ checked by BCA assay (Interchim, Montlucon, France).

\section{CE-MS analysis}

CE-MS analysis was performed as previously described $[37,47]$. The limit of detection was $\sim 1$ fmol, mass 
resolution was above 8000 enabling resolution of monoisotopic mass signals for $\mathrm{z} \leq 6$. After charge deconvolution, mass deviation was $<25$ ppm for monoisotopic resolution and $<100 \mathrm{ppm}$ for unresolved peaks $(\mathrm{z}>6)$. The analytical precision of the platform was assessed by (a) reproducibility achieved for repeated measurement of the same replicate and (b) by the reproducibility achieved for repeated preparation and measurement of the same urine sample; details on analytical precision were reported recently [21]. To ensure high data consistency, a minimum of 950 peptides/proteins had to be detected with a minimal MS resolution of 8,000 in a minimal migration time interval of 10 minutes.

\section{Data processing}

Mass spectral ion peaks representing identical molecules at different charge states were deconvoluted into single masses using MosaiquesVisu software [48]. Both CEmigration time and ion signal intensity (amplitude) show variability, mostly due to different concentration of ions in the sample, and are consequently normalized. Reference signals of 1770 urinary polypeptides are used for CE-time calibration by local regression. For normalization of analytical and urine dilution variances, MS signal intensities are normalized relative to 29 "housekeeping" peptides generally present in at least $90 \%$ of all urine samples with small relative standard deviation. For calibration, local regression is performed [49]. The obtained peak lists characterize each polypeptide by its molecular mass [Da], normalized CE migration time [min] and normalized signal intensity. All detected peptides were deposited, matched, and annotated in a Microsoft SQL database allowing further statistical analysis.

\section{Data analysis}

The datasets were examined either with respect to significant changes in single, predefined peptides and with respect to scoring in biomarker models (see Additional file 1, spreadsheet: 'classification factor'). These biomarker models consist of 65 or 273 biomarkers respectively, which were previously found to be significantly associates with diabetic nephropathy [19] or chronic kidney disease [20].

For the application of the previously established biomarker patterns, Wilcoxon test (for paired samples) was performed to receive Box-and-Whisker plots and dotand-line diagrams [50] (MedCalc version 8.1.1.0, MedCalc Software, Belgium, http://www.medcalc.be).

For multiple testing corrections, p-values were corrected using the false discovery rate procedure introduced by Benjamini and Hochberg, [51]. To eliminate sporadic findings, only proteins that were detected in a diagnostic group of patients in at least $50 \%$ of samples were considered.

\section{Results}

Samples from 22 patients included in the IRMA2 trial, where urine was collected at baseline before treatment (visit 2) and after two years treatment (visit 9) with Irbesartan or placebo, were analyzed. All available samples were included in the study, and analyzed using CE-MS, no additional specimens that fit the criteria (2 years follow up, placebo or $300 \mathrm{mg}$ Irbesartan daily) are available from the IRMA2 trial. All samples analyzed passed the threshold of the quality control criteria given in the Methods section, no significant deterioration of peptides due to storage could be observed. The data from all analyses are presented in the Additional file 1. As shown in figure 1 , the compiled data of these 4 groups disclosed first insights into changes of the urinary proteome, where high concentrations of some peptides decreased with Irbesartan intake. To assess the relevance of any proteomics changes with respect to diabetic nephropathy, we applied already established polypeptide patterns onto these data.

First, data from patients that received ARB treatment were evaluated applying a biomarker pattern indicative for diabetic nephropathy [19]. This analysis revealed no significant differences $(\mathrm{p}=0.175)$ between these two groups of patients (visit 2 and visit 9) using Wilcoxontest for paired samples (data not shown). However, the DN pattern was developed employing samples from diabetes type 1 patients treated with ARB [19], hence may not be applicable for type 2 diabetic patients, and may further be inappropriate to reflect drug-induced changes.

We therefore also employed a polypeptide pattern indicative of chronic kidney disease (CKD), that consist of 273 known peptides [20] for the classification of the urine samples from the 'IRMA2' study. This model is based on the CE-MS analysis of urine samples from 340 patients with CKD of different etiologies (including focal segmental glomerulosclerosis, membranous glomerulonephritis, minimal change disease, IgA nephropathy, systemic lupus erythematosus, ANCA-associated vasculitis, and diabetic nephropathy) and 550 controls (healthy individuals as well as patients without any evidence for renal diseases). Figure 2 demonstrates the changes of these 273 peptides of the CKD model before and after treatment with Irbesartan and placebo, respectively. While the peptide pattern of the ARB treated patients is similar to that observed for diabetic nephropathy (compare Figure 1 in [19]) at the beginning of the study (prior treatment), it changed towards higher similarity to normalbuminuric subjects after 2 years of Irbesartan 

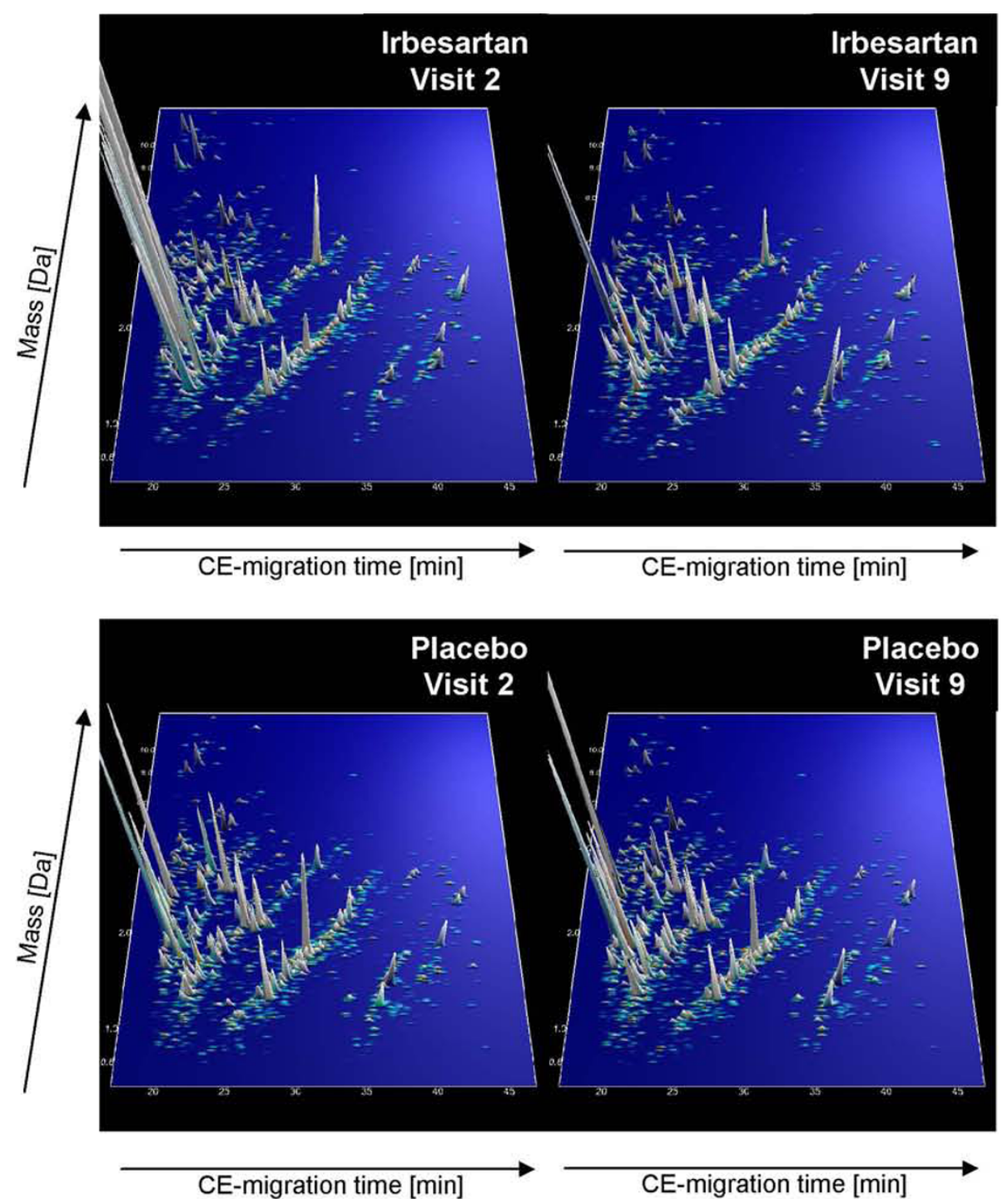

Figure 1 Polypeptide patterns of patients with diabetes type 2 before and after 2-year treatment (Irbesartan and placebo) examined in the 'IRMA2' study. Shown are compiled patterns consisting of all samples from each of the four groups. The molecular mass ( 0.7 to $15 \mathrm{kDa}$, on a logarithmic scale) is plotted against normalized migration time (17 to $47 \mathrm{~min}$ ). Signal intensity is encoded by peak height and color.

treatment. As depicted in the Box-and-Whisker plot in figure $3 \mathrm{~A}$, this classification resulted in a significant $(\mathrm{p}=0.0244)$ decline of the median classification factor (indicating an improvement of the kidney physiology), which was reduced (from 0.721 at visit 2 to 0.277 at visit 9) below the established cut-off (0.343) of the CKD model. Irrespective of the values before Irbesartan intake, the classification factors were decreasing during Irbesartan treatment in all patients except one (see figure $3 \mathrm{~B}$ ). This patient progressed to $\mathrm{DN}$ several years after the end of the study, none of the eleven patients developed macroalbuminuria during the two year study period. In the urine samples of the eleven patients treated with placebo, a non significant $(p=0.1016)$ increase (indicating a change towards "chronic kidney disease") of the median classification factor (see figure 3C) from -0.104 at visit 2 to 0.188 at visit 9 could be observed. Although many patients of the placebo-group scored lower than those of the Irbesartan-group at baseline before treatment (see figure 3B and 3D), the classification factor of most placebo-treated patients was higher after two years, as expected for progressing disease.

We subsequently investigated which of the 273 biomarkers that were found significantly associated with CKD undergo significant changes upon Irbesartan treatment. Eighteen of these CKD markers showed significant differences $(\mathrm{p}<0.05)$ in urine of patients before and after 2 year treatment with Irbesartan (see table 1). Of these 18, 

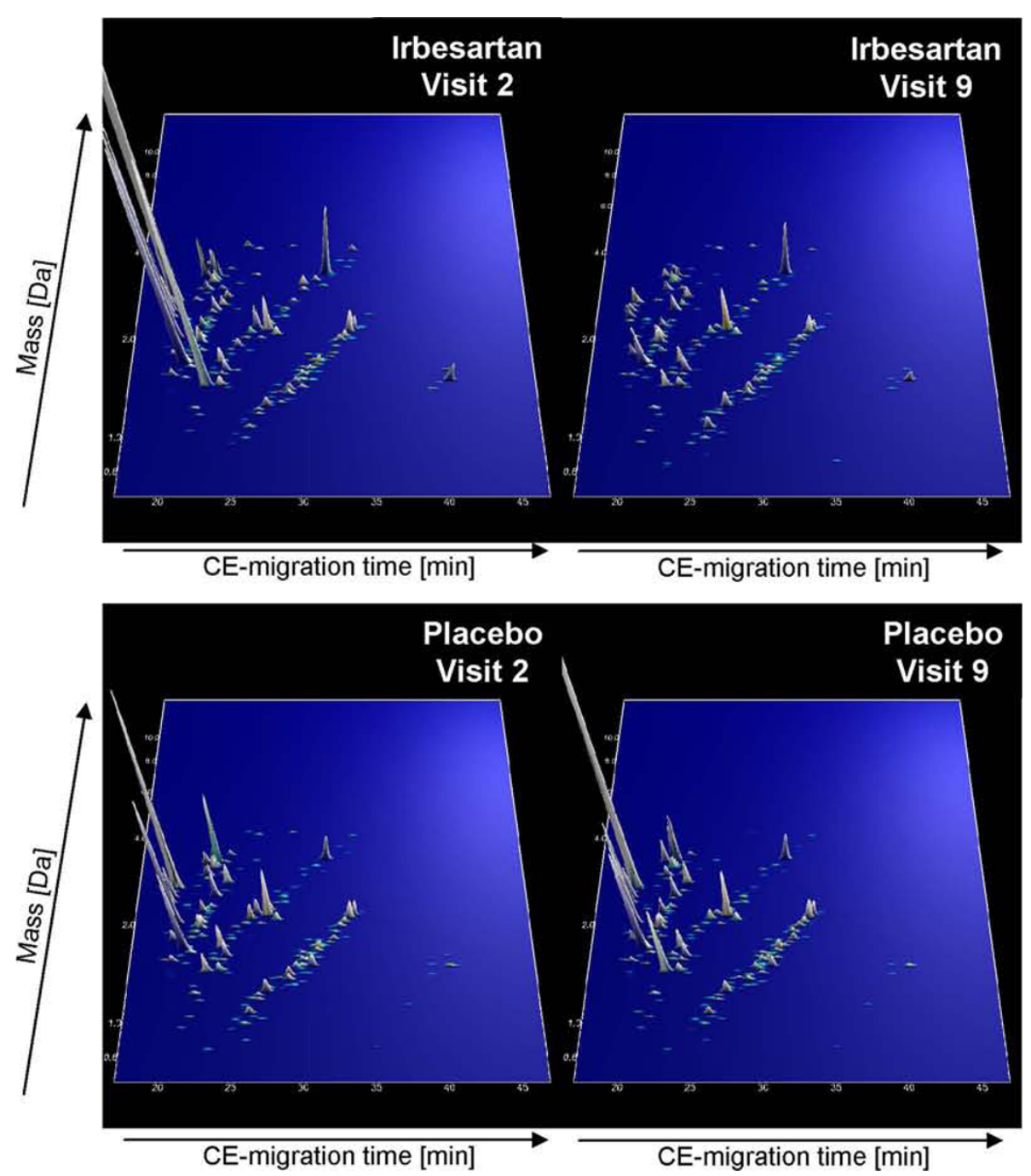

Figure 2 Peptide patterns of 273 CKD marker used for the proteomic analysis of patients from the 'IRMA2' subgroup. The compiled data sets of urine samples from patients derived from the 'IRMA2 study' before and after 2-year treatment of Irbesartan (upper panel) as well as placebo (lower panel) are shown. Normalized molecular mass ( $y$-axis) is plotted against normalized CE-migration time ( $x$-axis). The mean signal intensity is represented in 3D-depiction.

11 changed towards "normal controls", indicating possible benefit of therapy. Seven changes towards "chronic kidney disease", possibly indicating progression of pathophysiological changes over time that is not affected by therapy. We also investigated the 273 biomarkers in the placebo group. Here, we found 7 CKD markers which show significant differences within the 2-year treatment. Of these 7 peptides, all changed toward "disease". In total, 23 urinary CKD markers showed significant changes over the period of two years, either in the patients of the Irbesartan group or in the placebo group, 2 were significant in both groups. These two CKD markers, both collagen alpha-1 fragments (see table 1, bold letters), showed significant change towards "healthy" in the Irbesartan group and opposite regulation in the placebo group over the period of two years. While the amount of these two collagen fragments increased significantly in the ARB group (indicating an improvement towards "healthy"), their abundance was significantly decreased after 2 years of placebo treatment, indicating further progression of chronic kidney disease.

To obtain information on additional changes in the urinary proteome associated with Irbesatan treatment beyond those observed for the previously defined CKD biomarkers, we examined the data on all sequenced peptides $[40,52]$ for significant changes between baseline and 2-year treatment (in each group; Irbesartan and placebo). We could not identify additional biomarkers, which revealed significant changes between baseline and 2-year treatment. 

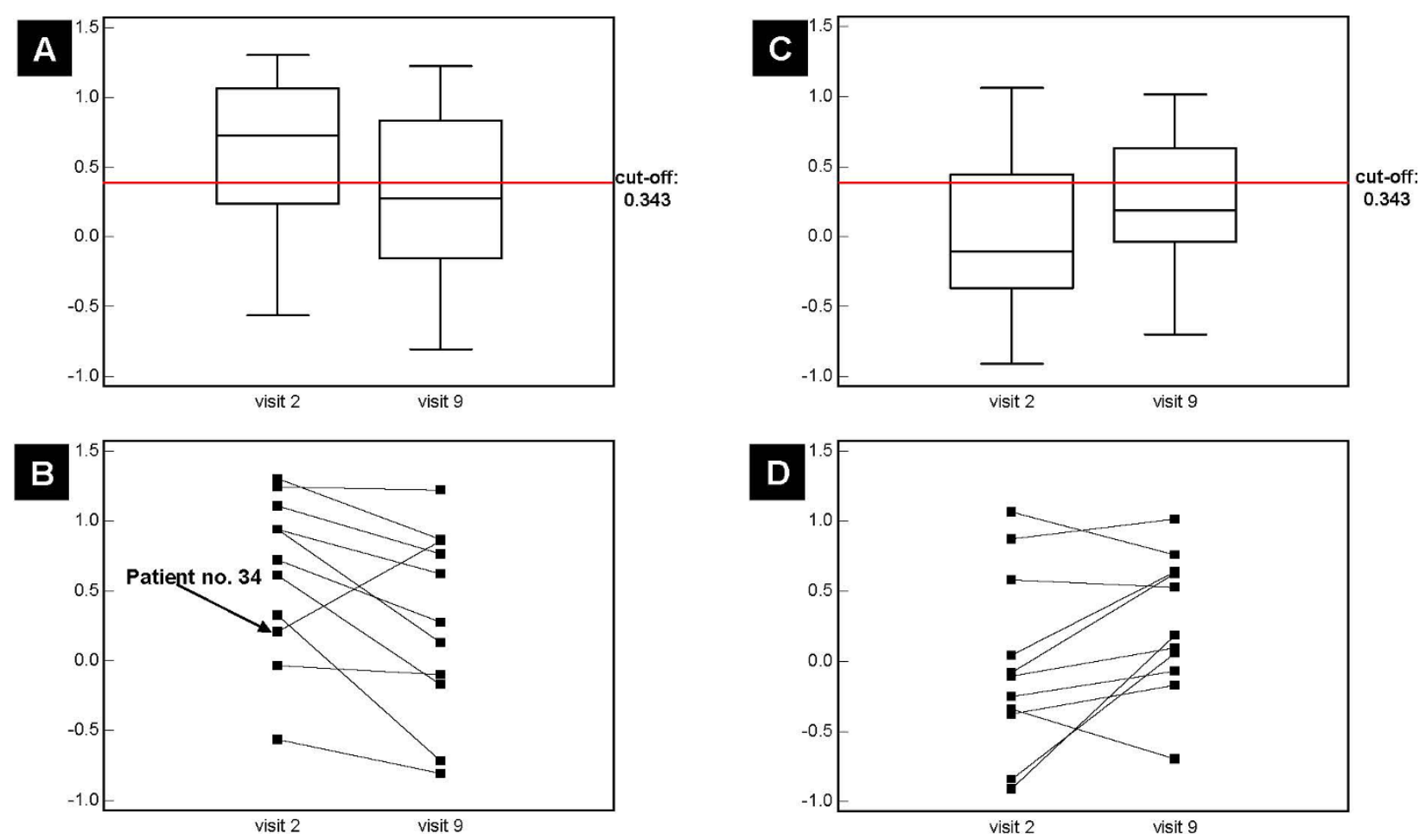

Figure 3 Classification results of the 'IRMA2' patient samples, classified with the CKD model [20]. A) Box-and-Whisker plot of microalbuminuric patients before (visit 2) and after two years (visit 9) treatment with $300 \mathrm{mg}$ Irbesartan. The red line indicates the cut-off of the CKD model (classification factors above this cut-off are suffering from renal disease). B) Dot-and-line diagram of microalbuminuric patients before (visit 2) and after two years (visit 9) treatment with 300 mg Irbesartan. Classification factors of all patients, excepting patient no. 34, declined after Irbesartan intake. C) Box-and-Whisker plot of microalbuminuric patients before (visit 2) and after two years (visit 9) treatment with placebo. D) Dot-and-line diagram of microalbuminuric patients before (visit 2) and after two years (visit 9) placebo administration.

\section{Discussion}

In the IRMA2 study a $300 \mathrm{mg}$ daily dose of the angiotensin II receptor blocker Irbesartan significantly reduced albuminuria compared to placebo [6]. Using CE-MS analysis of urine in all available samples (a subset of 22 of these patients) we were able to demonstrate a persistent and significant changes of the previously established proteomic CKD classifier [20] towards "healthy". After long-term renoprotective treatment with Irbesartan. Furthermore, the proteomic analysis of placebo treated patients showed a slight, yet not significant, increase of this classifier. This increase likely reflects disease progression in the absence of appropriate therapy, like blocking the renin angiotensin system demonstrated to protect against development of diabetic nephropathy.

We have previously reported that collagen fragments are reduced in patients with diabetic nephropathy [19]. After confirmation in additional samples, we generated the hypothesis that this reduction in urinary collagen fragments may be an indicator of attenuated collagen breakdown, resulting in fibrosis [53]. The results presented here further indicate that this process may be positively influenced by ARB treatment, resulting in an increase in urinary collagen fragments, likely reflecting an increase of proteolysis towards normal ("healthy") physiological levels. It is tempting to speculate that the urinary proteomic changes observed here may be a consequence of an actual change in renal pathophysiology, and not merely a consequence of the changes in urine protein concentration. To substantiate this hypothesis, analysis of longitudinal samples on a larger cohort will be undertaken.

As we also could show recently, the collagen fragments have similar quality as biomarkers in both, $24 \mathrm{~h}$ and spot urine [49]. This is to be expected since their secretion into urine does not appear to change significantly during the day (Mischak, unpublished), and the concentration of these biomarkers is assessed in reference to internal standards, in a similar way as albumin/ creatinine ratio.

The changes in the urinary proteome reported here were observed employing a biomarker pattern that is associated with CKD in general, not restricted to 
Table 1 Significance analysis of CKD markers in urine of microalbuminuric patients before and after two year treatment

\begin{tabular}{|c|c|c|c|c|c|c|}
\hline $\begin{array}{l}\text { CKD } \\
\text { marker }\end{array}$ & Sequence & Peptide name & $\begin{array}{l}\text { Wilcoxon } \\
\text { p-value } \\
\text { (Irbesaran } \\
\text { treatment) }\end{array}$ & $\begin{array}{c}\text { Wilcoxon } \\
\text { p-value } \\
\text { (Placebo } \\
\text { intake) }\end{array}$ & $\begin{array}{l}\text { Irbesartan } \\
\text { treatment }\end{array}$ & $\begin{array}{l}\text { Placebo } \\
\text { intake }\end{array}$ \\
\hline 2505 & SpGEAGRpG & Collagen alpha-1 (I) chain [522-530] & $1.58 \mathrm{E}-02$ & n.s. & $\uparrow$ & - \\
\hline 3508 & GPpGPpGPpG & Collagen alpha-1 (I) chain [145-154] & $1.58 \mathrm{E}-02$ & n.s. & $\uparrow$ & - \\
\hline 11982 & YQTNKAKH & Cystatin-B [85-92] & $2.55 \mathrm{E}-03$ & n.s. & $\downarrow$ & - \\
\hline 13342 & ApGDKGESGPS & Collagen alpha-1 (I) chain [777-787] & $4.46 \mathrm{E}-02$ & $8.33 \mathrm{E}-03$ & $\uparrow$ & $\downarrow$ \\
\hline 14906 & MGPRGPpGPpG & Collagen alpha-1 (I) chain [217-227] & n.s. & $1.94 \mathrm{E}-02$ & - & $\downarrow$ \\
\hline 15800 & GEYKFQNAL & $\begin{array}{l}\text { Serum albumin } \\
{[423-431]}\end{array}$ & $1.53 \mathrm{E}-03$ & n.s. & $\uparrow$ & - \\
\hline 17694 & ApGDRGEpGPp & Collagen alpha-1 (I) chain [798-808] & 5.39E-05 & n.s. & $\uparrow$ & - \\
\hline 24117 & SpGPDGKTGPPGp & Collagen alpha-1 (I) chain [546-558] & $3.02 \mathrm{E}-02$ & $1.58 \mathrm{E}-02$ & $\uparrow$ & $\downarrow$ \\
\hline 24958 & GPpGPDGNKGEpG & Collagen alpha-2 (I) chain [613-625] & $1.84 \mathrm{E}-02$ & n.s. & $\uparrow$ & - \\
\hline 25053 & GPpGEAGKpGEQG & Collagen alpha-1 (I) chain [650-662] & $1.25 \mathrm{E}-03$ & n.s. & $\uparrow$ & - \\
\hline 28747 & SpGERGETGPpGP & Collagen alpha-1 (III) chain [796-808] & 4.10E-03 & n.s. & $\uparrow$ & - \\
\hline 38780 & GLpGTGGPpGENGKpG & Collagen alpha-1 (III) chain [642-657] & n.s. & $2.62 \mathrm{E}-02$ & - & $\downarrow$ \\
\hline 55523 & SpGSNGApGQRGEpGPQG & Collagen alpha-1 (III) chain [358-375] & n.s. & 4.47E-02 & - & $\downarrow$ \\
\hline 61573 & DEAGSEADHEGTHSTKR & Fibrinogen alpha chain $[605-621]$ & $1.92 \mathrm{E}-02$ & n.s. & $\uparrow$ & - \\
\hline 73177 & DAGApGAPGGKGDAGApGERGPpG & Collagen alpha-1 (III) chain [664-687] & 7.81E-03 & n.s. & $\downarrow$ & - \\
\hline 73697 & GNSGEpGApGSKGDTGAKGEPGp & Collagen alpha-1 (I) chain [431-453] & n.s. & $3.51 \mathrm{E}-02$ & - & $\downarrow$ \\
\hline 78332 & AGPpGEAGKpGEQGVpGDLGAPGP & Collagen alpha-1 (I) chain [646-669] & $1.04 \mathrm{E}-02$ & n.s. & $\downarrow$ & - \\
\hline 81196 & NGApGNDGAkGDAGApGAPGSQGApG & Collagen alpha-1 (I) chain [700-725] & 2.33E-02 & n.s. & $\downarrow$ & - \\
\hline 82026 & GNSGEpGApGSKGDTGAKGEpGPVG & Collagen alpha-1 (I) chain [431-455] & $2.71 \mathrm{E}-02$ & n.s. & $\downarrow$ & - \\
\hline 94308 & TGPIGPpGPAGApGDKGESGPSGPAGPTG & Collagen alpha-1 (I) chain [766-794] & 1.30E-02 & n.s. & $\downarrow$ & - \\
\hline 96370 & LmIEQNTKSPLFMGKWNPTQK & Alpha-1-antitrypsin [397-418] & 4.47E-02 & n.s. & $\uparrow$ & - \\
\hline 118224 & ESGREGApGAEGSpGRDGSpGAKGDRGETGPA & Collagen alpha-1 (I) chain [1011-1042] & n.s. & $6.94 \mathrm{E}-03$ & - & $\downarrow$ \\
\hline 143947 & $\begin{array}{l}\text { DQGPVGRTGEVGAVGPpGFAGEKGPSG } \\
\text { EAGTAGPpGTpGPQG }\end{array}$ & Collagen alpha-2 (I) chain [824-865] & $1.59 \mathrm{E}-03$ & n.s. & $\downarrow$ & - \\
\hline
\end{tabular}

Wilcoxon p-values $(\mathrm{p}<0.05)$ of CKD markers, which show changes in the comparison of patients urine before and after longterm treatment are listed. In addition, sequences and peptide names of the significant markers are shown ( $\mathrm{p}=$ hydroxyproline, $\mathrm{k}=\mathrm{hydroxylysine,} \mathrm{m}=\mathrm{oxidation}$ of methionine). In the last three columns the regulation of those markers is depicted. Arrow upwards indicates significant change towards "healthy"; arrow pointing down indicates significant change towards "CKD".

diabetic nephropathy. This observation indicates that analysis of changes in the urinary proteome may also be useful in evaluation of treatments for other forms of kidney disease. Of note, drug-induced changes in the urinary proteome indicating benefit of therapy were recently reported for ANCA-associated vasculitis [21]. While the data currently available cannot clarify this issue, further analysis of urine samples from other therapeutic trials involving different drugs and other diseases (glomerulosclerosis and IgA Nephropathy) are planned. These may help to further support this hypothesis.

A shortcoming of the study reported here is the relatively low number of patients included. Unfortunately, no additional samples are available from the IRMA2 trial, hence this cannot be improved upon. However, the results were very consistent within each group. Even more relevant, we demonstrate on a very low number of only 11 treated and 11 untreated subjects, that ARB treatment does have a statistically significant positive effect, based on the proteomic CKD biomarker pattern, hence we feel that the report is in agreement with the recently published guidelines for proteomic biomarkers [54]. While we cannot exclude the presence of other confounders or underlying bias, we have no indication that confounders like e.g. drugs or infectious diseases at the time of sampling had a significance impact.

The results highlight an advantage of the urinary proteome analysis: a small number of subjects included in a trial may be sufficient to reveal significant effects of drug treatment, based on a classifier that serves as a surrogate marker. While such data can currently not replace hard endpoints like ESRD, they may serve to give guidance, e.g. for the decision if a drug may be likely to exert a positive influence on disease/disease progression. 


\section{Conclusion}

The data introduce urinary proteome analysis as a novel method not only for assessment of new drugs and therapeutic regimens in CKD, but also for the treatment monitoring of patients on renoprotective drugs. Furthermore, the data strengthen the hypothesis that collagens play an important role in the development of diabetic nephropathy (see also [53]) and that collagen turnover may be a highly suitable target for diagnosis and novel therapeutic approaches of this disease.

The proteomic biomarker pattern employed here (the CKD-273 pattern [20]) may well be a superior surrogate in comparison to the frequently used assessment of urinary albumin. To test this hypothesis, UAE and proteomic patterns from samples of longitudinal studies that reach hard endpoints have to be compared.

\section{Additional material}

Additional file 1: Raw data and additional information. Table consists of 4 different spreadsheets called patients data, classification factor, polypeptides, and patient's raw data. Patients data. This table lists information of each patient, including patients IDs and treatment. Furthermore urinary albumin concentration, eGFR, and blood pressure are given at baseline and after two years. Classification factors. Table show the classification factors of all measured urine samples, including patients IDs, sample ID, and treatment. Polypeptides. Table listing 2,044 different peptides/proteins (Protein ID) detected, their calibrated molecular mass [Da], and normalized CE migration time [min]. Furthermore, sequence information is given, if available. Patient's raw data. Tables in pivot format show the CE-MS raw data of the 44 samples in the database. The protein IDs of all peptides are given in the first column named "Protein ID"; the unique patients IDs constitute the first row. The MS data from each sample are reflected in one column. The number in each cell represents the calibrated amplitude of the mass spectrometric signal of each peptide/protein detected in the sample.

\section{Abbreviations}

ARB: angiotensin II receptor blocker; CE-MS: capillary electrophoresis couples to mass spectrometry; CKD: chronic kidney disease; ESRD: end-stage renal disease; UAE: urinary albumin excretion; RAAS: renin-angiotensin-aldosterone system; IRMA-2: Irbesartan in Patients with Type 2 Diabetes and Microalbuminuria Study

\section{Acknowledgements}

This study was carried out with financial support of the Commission of the European Communities, $6^{\text {th }}$ Framework Programme Priority 1, Life Sciences, Genomics and Biotechnology for Health, LSHM-CT-2005-018733, acronym PREDICTIONS (PREvention of Dlabetic ComplicaTIONS) and the $7^{\text {th }}$ Framework Programme, grant agreement HEALTH-F2-2009-241544 (SysKID).

\section{Author details}

${ }^{1}$ Steno Diabetes Centre, Gentofte, Denmark. ${ }^{2}$ BHF Glasgow Cardiovascular Research Centre, Glasgow, United Kingdom. ${ }^{3}$ Mosaiques diagnostics GmbH, Hannover, Germany. ${ }^{4}$ Department of Medical Endocrinology Rigshospitalet, University Hospital of Copenhagen, Copenhagen Denmark. ${ }^{5}$ Faculty of Health Sciences, University of Aarhus, Aarhus, Denmark. ${ }^{6}$ members of EuroKUP.

\section{Authors' contributions}

SA participated in the design of the study and performed the statistical analysis. PZ and HM performed the CE-MS analysis and data evaluation. $\mathrm{H}$ -
HP and PR conceived of the study, and participated in its design and coordination. All authors were involved in drafting the manuscript, have read and approved the final manuscript.

\section{Competing interests}

Harald Mischak is co-founder and a co-owner of mosaiques diagnostics \& therapeutics AG, (Hannover, Germany). Petra Zürbig is an employee of mosaiques diagnostics $\mathrm{GmbH}$. Peter Rossing has received speakers honorarium from Novartis, Sanofi-Aventis, Boehringer Ingelheim, and MSD, and research grants from Novartis. Hans-Henrik Parving has received speakers honorarium from Novartis and consulting fees from Novartis.

Received: 1 July 2010 Accepted: 1 November 2010

Published: 1 November 2010

\section{References}

1. Wild S, Roglic G, Green A, Sicree R, King H: Global prevalence of diabetes: estimates for the year 2000 and projections for 2030. Diabetes Care 2004, 27:1047-1053.

2. Parving HH, Mauer M, Ritz E: Diabetic nephropathy. In Brenner and Rector's the Kidney. Edited by: Brenner BM. Philadelphia: WB Saunders; 2004:1777-1818.

3. Mogensen CE: Microalbuminuria predicts clinical proteinuria and early mortality in maturity-onset diabetes. N Engl J Med 1984, 310:356-360.

4. de Zeeuw D, Remuzzi G, Parving HH, Keane WF, Zhang Z, Shahinfar S, et al: Albuminuria, a therapeutic target for cardiovascular protection in type 2 diabetic patients with nephropathy. Circulation 2004, 110:921-927.

5. Brenner BM, Cooper ME, de Zeeuw D, Keane WF, Mitch WE, Parving $\mathrm{H}-\mathrm{H}$ : Effects of Losartan on Renal and Cardiovascular outcomes in patients with type 2 diabetes and nephropathy. N Engl J Med 2001, 345:861-869.

6. Parving HH, Lehnert $\mathrm{H}$, Brochner-Mortensen J, Gomis R, Andersen S, Arner P: The effect of irbesartan on the development of diabetic nephropathy in patients with type 2 diabetes. N Engl J Med 2001, 345:870-878

7. Lewis EJ, Hunsicker LG, Clarke WR, Berl T, Pohl MA, Lewis JB, et al: Renoprotective effect of the angiotensin-receptor antagonist irbesartan in patients with nephropathy due to type 2 diabetes. N Engl J Med 2001, 345:851-860

8. American Diabetes Association: Diabetic Nephropathy. Diabetes Care 2002, 25:85-89.

9. Mischak H, Kaiser T, Walden M, Hillmann M, Wittke S, Herrmann A, et al: Proteomic analysis for the assessment of diabetic renal damage in humans. Clin Sci (Lond) 2004, 107:485-495

10. Meier M, Kaiser T, Herrmann A, Knueppel S, Hillmann M, Koester $P$, et al Identification of urinary protein pattern in type 1 diabetic adolescents with early diabetic nephropathy by a novel combined proteome analysis. J Diabetes Complications 2005, 19:223-232.

11. Rossing $\mathrm{K}$, Mischak $\mathrm{H}$, Parving HH, Christensen PK, Walden M, Hillmann M, et al: Impact of diabetic nephropathy and angiotensin II receptor blockade on urinary polypeptide patterns. Kidney Int 2005, 68:193-205.

12. Fliser D, Novak J, Thongboonkerd V, Argiles A, Jankowski V, Girolami M, et al: Advances in urinary proteome analysis and biomarker discovery. $J$ Am Soc Nephrol 2007, 18:1057-1071.

13. Julian BA, Wittke S, Novak J, Good DM, Coon JJ, Kellmann M, et al: Electrophoretic methods for analysis of urinary polypeptides in IgAassociated renal diseases. Electrophoresis 2007, 28:4469-4483.

14. Kistler AD, Mischak H, Poster D, Dakna M, Wuthrich RP, Serra AL: Identification of a unique urinary biomarker profile in patients with autosomal dominant polycystic kidney disease. Kidney Int 2009, 76:89-96.

15. Drube J, Schiffer E, Mischak H, Kemper MJ, Neuhaus T, Pape L, et al: Urinary proteome pattern in children with renal Fanconi syndrome. Nephrol Dial Transplant 2009, 24:2161-2169.

16. Candiano G, Musante L, Bruschi M, Petretto A, Santucci L, Del BP, et al: Repetitive fragmentation products of albumin and alpha1-antitrypsin in glomerular diseases associated with nephrotic syndrome. J Am SoC Nephrol 2006, 17:3139-3148.

17. Nguyen MT, Ross GF, Dent CL, Devarajan P: Early prediction of acute renal injury using urinary proteomics. Am J Nephrol 2005, 25:318-326.

18. Dihazi H, Muller GA, Lindner S, Meyer M, Asif AR, Oellerich $M$, et al: Characterization of diabetic nephropathy by urinary proteomic analysis: 
identification of a processed ubiquitin form as a differentially excreted protein in diabetic nephropathy patients. Clin Chem 2007, 53:1636-1645.

19. Rossing K, Mischak H, Dakna M, Zürbig P, Novak J, Julian BA, et al: Urinary proteomics in diabetes and CKD. J Am Soc Nephrol 2008, 19:1283-1290.

20. Good DM, Zurbig P, Argiles A, Bauer HW, Behrens G, Coon JJ, et al: Naturally occurring human urinary peptides for use in diagnosis of chronic kidney disease. Mol Cell Proteomics 2010.

21. Haubitz M, Good DM, Woywodt A, Haller H, Rupprecht H, Theodorescu D, et al: Identification and Validation of Urinary Biomarkers for Differential Diagnosis and Evaluation of Therapeutic Intervention in ANCA associated Vasculitis. Mol Cell Proteomics 2009, 8:2296-2307.

22. Alkhalaf A, Zuerbig P, Bakker SJL, Bilo HJ, Cerna M, Fischer C, et al: Multicentric validation of pproteomic biomarkers in urine specific for diabetic nephropathy. PLOS ONE 2010.

23. Mischak H, Rossing P: Proteomic Biomarkers in diabetic nephropathy reality or future promise? Nephrol Dial Transplant 2010.

24. Ameur RB, Molina L, Bolvin C, Kifagi C, Jarraya F, Ayadi H, et al: Proteomic approaches for discovering biomarkers of diabetic nephropathy. Nephrol Dial Transplant 2010

25. Vidal BC, Bonventre JV, Hong HS: Towards the application of proteomics in renal disease diagnosis. Clin Sci (Lond) 2005, 109:421-430.

26. Decramer S, Gonzalez de PA, Breuil B, Mischak H, Monsarrat B, Bascands JL, et al: Urine in clinical proteomics. Mol Cell Proteomics 2008, 7:1850-1862.

27. Molina F, Dehmer M, Perco P, Graber A, Girolami M, Spasovski G, et al: Systems biology: opening new avenues in clinical research. Nephrol Dial Transplant 2010, 25(4):1015-8.

28. Dominiczak AF, Herget-Rosenthal S, Delles C, Fliser D, Fournier I, Graber A, et al: Systems biology to battle vascular disease. Nephrol Dial Transplant 2010, 25(4):1019-22.

29. Zhou H, Pisitkun T, Aponte A, Yuen PS, Hoffert JD, Yasuda H, et al: Exosomal Fetuin-A identified by proteomics: A novel urinary biomarker for detecting acute kidney injury. Kidney Int 2006, 70(10):1847-57.

30. Pieper R, Gatlin CL, McGrath AM, Makusky AJ, Mondal M, Seonarain M, et al: Characterization of the human urinary proteome: A method for highresolution display of urinary proteins on two-dimensional electrophoresis gels with a yield of nearly 1400 distinct protein spots. Proteomics 2004, 4:1159-1174.

31. Thongboonkerd V, McLeish KR, Arthur JM, Klein JB: Proteomic analysis of normal human urinary proteins isolated by acetone precipitation or ultracentrifugation. Kidney Int 2002, 62:1461-1469.

32. Pisitkun T, Shen RF, Knepper MA: Identification and proteomic profiling of exosomes in human urine. Proc Natl Acad Sci USA 2004, 101:13368-13373.

33. Marshall $\mathrm{T}$, Williams $\mathrm{K}$ : Two-dimensional electrophoresis of human urinary proteins following concentration by dye precipitation. Electrophoresis 1996, 17:1265-1272.

34. Shihabi ZK, Konen JC, O'Connor ML: Albuminuria vs urinary total protein for detecting chronic renal disorders. Clin Chem 1991, 37:621-624.

35. Yudkin JS, Forrest RD, Jackson CA: Microalbuminuria as predictor of vascular disease in non-diabetic subjects. Islington Diabetes Survey. Lancet 1988, 2:530-533.

36. Schaub S, Rush D, Wilkins J, Gibson IW, Weiler T, Sangster K, et al: Proteomic-based detection of urine proteins associated with acute renal allograft rejection. J Am Soc Nephrol 2004, 15:219-227.

37. Kolch W, Neususs C, Pelzing M, Mischak H: Capillary electrophoresis-mass spectrometry as a powerful tool in clinical diagnosis and biomarker discovery. Mass Spectrom Rev 2005, 24:959-977.

38. Good DM, Thongboonkerd V, Novak J, Bascands JL, Schanstra JP, Coon JJ, et al: Body fluid proteomics for biomarker discovery: lessons from the past hold the key to success in the future. J Proteome Res 2007, 6:4549-4555

39. Schaub S, Wilkins J, Weiler T, Sangster K, Rush D, Nickerson P: Urine protein profiling with surface-enhanced laser-desorption/ionization time-of-flight mass spectrometry. Kidney Int 2004, 65:323-332.

40. Mischak H, Kolch W, Aivalotis M, Bouyssie D, Court M, Dihazi H, et al: Comprehensive human urine standards for comparability and standardization in clinical proteome analysis. Proteomics Clin Appl 2010, 4:464-478.

41. Haubitz M, Wittke S, Weissinger EM, Walden M, Rupprecht HD, Floege J, et al: Urine protein patterns can serve as diagnostic tools in patients with IgA nephropathy. Kidney Int 2005, 67:2313-2320.
42. Weissinger EM, Wittke $\mathrm{S}$, Kaiser $\mathrm{T}$, Haller $\mathrm{H}$, Bartel $\mathrm{S}$, Krebs R, et al: Proteomic patterns established with capillary electrophoresis and mass spectrometry for diagnostic purposes. Kidney Int 2004, 65:2426-2434.

43. Theodorescu D, Fliser D, Wittke S, Mischak H, Krebs R, Walden M, et al: Pilot study of capillary electrophoresis coupled to mass spectrometry as a tool to define potential prostate cancer biomarkers in urine. Electrophoresis 2005, 26:2797-2808.

44. Snell-Bergeon JK, Maahs DM, Ogden LG, Kinney GL, Hokanson JE, Schiffer E, et al: Evaluation of urinary biomarkers for coronary artery disease, diabetes, and diabetic kidney disease. Diabetes Technol Ther 2009, 11:1-9.

45. Andersen S, Brochner-Mortensen J, Parving HH: Kidney function during and after withdrawal of long-term irbesartan treatment in patients with type 2 diabetes and microalbuminuria. Diabetes Care 2003, 26:3296-3302.

46. Zürbig P, Renfrow MB, Schiffer E, Novak J, Walden M, Wittke S, et al: Biomarker discovery by CE-MS enables sequence analysis via MS/MS with platform-independent separation. Electrophoresis 2006, 27:2111-2125.

47. Theodorescu D, Wittke S, Ross MM, Walden M, Conaway M, Just I, et al: Discovery and validation of new protein biomarkers for urothelial cancer: a prospective analysis. Lancet Oncol 2006, 7:230-240.

48. Neuhoff N, Kaiser T, Wittke S, Krebs R, Pitt A, Burchard A, et al: Mass spectrometry for the detection of differentially expressed proteins: a comparison of surface-enhanced laser desorption/ionization and capillary electrophoresis/mass spectrometry. Rapid Communications in Mass Spectrometry 2004, 18:149-156.

49. Jantos-Siwy J, Schiffer E, Brand K, Schumann G, Rossing K, Delles C, et al: Quantitative Urinary Proteome Analysis for Biomarker Evaluation in Chronic Kidney Disease. J Proteome Res 2009, 8:268-281.

50. DeLeo JM: DeLeo, J.M. Receiver operating characteristic laboratory (ROCLAB): Software for developing decision strategies that account for uncertainty. College Park, MD, USA; 1993, 318-325.

51. Benjamini $Y$, Hochberg $Y$ : Controlling the false discovery rate: a practical and powerful approach to multiple testing. I Royal Stat Soc $B$ (Methodological) 1995, 57:125-133.

52. Coon JJ, Zürbig P, Dakna M, Dominiczak AF, Decramer S, Fliser D, et al: CEMS analysis of the human urinary proteome for biomarker discovery and disease diagnostics. Proteomics Clin Appl 2008, 2:964-973.

53. Rossing K, Mischak H, Rossing P, Schanstra JP, Wiseman A, Maahs DM: The urinary proteome in diabetes and diabetes-associated complications: new ways to assess disease progression and evaluate therapy. Proteomics Clin Appl 2008, 2:997-1007.

54. Mischak H, Allmaier G, Apweiler R, Attwood T, Baumann M, Benigni A, et al: Recommendations for biomarker identification and qualification in clinical proteomics. Sci Transl Med 2010, 2:46ps42.

\section{Pre-publication history}

The pre-publication history for this paper can be accessed here: http://www.biomedcentral.com/1471-2369/11/29/prepub

doi:10.1186/1471-2369-11-29

Cite this article as: Andersen et al: Urinary proteome analysis enables assessment of renoprotective treatment in type 2 diabetic patients with microalbuminuria. BMC Nephrology 2010 11:29.

\section{Submit your next manuscript to BioMed Central and take full advantage of:}

- Convenient online submission

- Thorough peer review

- No space constraints or color figure charges

- Immediate publication on acceptance

- Inclusion in PubMed, CAS, Scopus and Google Scholar

- Research which is freely available for redistribution 